\title{
Eltrombopag-induced liver dysfunction during the treatment of immune thrombocytopenia and its risk factors
}

\author{
Ping Zhang, Wenjuan Miao
}

State Key Laboratory of Experimental Hematology, National Clinical Research Center for Blood Diseases, Institute of Hematology \& Blood Diseases Hospital, Chinese Academy of Medical Sciences \& Peking Union Medical College, Tianjin, China

Contributions: (I) Conception and design: P Zhang; (II) Administrative support: P Zhang; (III) Provision of study materials or patients: P Zhang; (IV) Collection and assembly of data: W Miao; (V) Data analysis and interpretation: W Miao; (VI) Manuscript writing: Both authors; (VII) Final approval of manuscript: Both authors.

Correspondence to: Ping Zhang. Institute of Hematology and Blood Diseases Hospital, Chinese Academy of Medical Sciences, State Key Laboratory of Experimental Hematology, National Clinical Research Center for Blood Diseases, Tianjin, China. Email: zhangping@ihcams.ac.cn.

Background Eltrombopag is an effective oral thrombopoietin receptor agonist for the treatment of immune thrombocytopenia (ITP). A common adverse reaction is liver dysfunction. This study aimed to investigate early liver dysfunction during eltrombopag treatment of ITP and risk factors.

Methods: We retrospectively analyzed liver dysfunction in patients receiving eltrombopag at the Blood Diseases Hospital, Chinese Academy of Medical Sciences, between September 2019 and December 2020. Patients were divided into two groups, those with and without liver dysfunction after eltrombopag treatment. Clinical characteristics of the two groups of patients, including sex, age, body mass index (BMI), comorbidities, concomitant medications, prophylactic use of hepatoprotective drugs, and eltrombopag usage were analyzed to identify the risk factors of liver dysfunction.

Results: A total of 85 patients were included in this study, including 28 men and 57 women aged $44 \pm 17$ years with a BMI of $25.1 \pm 3.57 \mathrm{~kg} / \mathrm{m}^{2}$. After eltrombopag treatment, liver dysfunction occurred in 19 patients $(22.4 \%)$, mainly in the form of elevated alanine aminotransferase (ALT; $\mathrm{n}=10,11.8 \%$ ) and total blood bilirubin (TBIL; $\mathrm{n}=11,12.9 \%$ ). Among patients with liver dysfunction, 73.7\% had grade 1, 15.8\% had grade 2 , and $10.5 \%$ had grade 3 . There were 7 cases (8.2\%) of drug-induced hepatocellular injury, of which 6 cases $(85.7 \%)$ were mild and 1 (14.3\%) was moderate. No significant between-group difference was observed in sex, age, BMI, use of other drugs that can induce liver dysfunction, prophylactic use of hepatoprotective drugs, or initial dose, usage time, or cumulative dose of eltrombopag (all $\mathrm{P}>0.05$ ). Comorbid type 2 diabetes or noninflammatory hepatobiliary disease (gallbladder stones, gallbladder polyps, liver cysts, fatty liver) was significantly correlated with liver dysfunction $(\mathrm{P}=0.04, \mathrm{P}=0.023$, respectively).

Conclusions: The risk of early liver dysfunction, while mild to moderate in most cases, is high in patients with ITP on eltrombopag treatment, especially in those with type 2 diabetes and hepatobiliary diseases. Therefore, patients should be closely monitored during treatment to enable timely intervention as needed.

Keywords: Thrombopoietin receptor agonist; eltrombopag; immune thrombocytopenia (ITP); liver dysfunction; drug-induced liver injury; risk factors

Submitted Mar 08, 2021. Accepted for publication Jun 04, 2021.

doi: 10.21037/apm-21-1067

View this article at: https://dx.doi.org/10.21037/apm-21-1067 


\section{Introduction}

Immune thrombocytopenia (ITP) is an acquired autoimmune bleeding disease characterized by isolated peripheral blood platelet count reduction (1). The main pathogenesis of ITP is the loss of immune tolerance of platelet autoantigens, which leads to abnormal activation of humoral and cellular immunity, and therefore mediate the acceleration of platelet destruction and the lack of platelet production by megakaryocytes. The treatment of ITP follows the principle of individualization, aiming to increase the platelet count to a safe level on the basis of minimizing the adverse effects of the treatment, reduce bleeding events, and pay attention to the health-related quality of life of the patient. Eltrombopag is an oral nonpeptide thrombopoietin (TPO) receptor agonist (2) that binds to the myeloproliferative leukemia transmembrane domain of TPO receptor to activate the Janus kinase/signal transducer and activator of transcription (JAK/STAT) signaling pathway, thereby stimulating megakaryocyte proliferation and differentiation and promoting platelet production (3). In July 2018, eltrombopag olamine (Revolade) was the first TPO receptor agonist approved by the China Food and Drug Administration to treat adults and children aged 12 or above with ITP who do not respond to glucocorticoids or immunoglobulins. The Chinese guidelines on the diagnosis and management of adult primary immune thrombocytopenia (version 2020) (1) state that eltrombopag may be used as second-line treatment for primary ITP in adults, in whom it has a response rate of $60 \%$ or more. Furthermore, eltrombopag has been authorized for use in pediatric patients with chronic ITP by the US Food and Drug Administration (FDA), and the study of eltrombopag in the treatment of Chinese children with ITP not only shows excellent initial response but also has continued efficacy and safety (4). Several studies (5-7) have shown that eltrombopag olamine is well tolerated under long-term use, with mild adverse reactions and rarely serious adverse reactions (8). A common adverse reaction is liver dysfunction, as manifested in elevated alanine aminotransferase (ALT) or total blood bilirubin (TBIL). Both US Food and Drug Administration instructions for use and Chinese package inserts have included warnings that eltrombopag may increase the risk of serious or potentially life-threatening liver toxicity. Therefore, early detection of liver dysfunction and identification of high-risk populations are essential for the wide use of eltrombopag. In this study, we retrospectively analyzed the clinical data of ITP patients who received eltrombopag at the Blood Diseases Hospital of the Chinese Academy of Medical Sciences between September 2019 and December 2020 to investigate liver dysfunction and its risk factors.

We present the following article in accordance with the STROBE reporting checklist (available at http://dx.doi. org/10.21037/apm-21-1067).

\section{Methods}

\section{Subjects}

The research protocol was approved by the Ethics Committee, Institute of Hematology and Blood Diseases Hospital, Chinese Academy of Medical Sciences (HG2021008-EC-1). All procedures performed in this study involving human participants were in accordance with the Declaration of Helsinki (as revised in 2013). Individual consent for this retrospective analysis was waived. Patients receiving eltrombopag olamine (Revolade) at our hospital between September 2019 and December 2020 were included in this study if they met the following inclusion criteria: (I) age at least 12 years as per the "Indications" on the eltrombopag olamine package insert; (II) ITP diagnosed based on the Chinese guideline on the diagnosis and management of adult primary immune thrombocytopenia (version 2020) (1); (III) eltrombopag treatment for at least 5 days at our hospital; and (IV) complete liver monitoring data before and after eltrombopag treatment. Exclusion criteria: (I) <12 years of age; (II) no diagnosis of ITP; (III) history of hepatitis; (IV) eltrombopag treatment $<5$ days; or (V) incomplete clinical data.

\section{Methods}

We collected the electronic medical records of all eligible patients via the hospital information platform. Patients were divided into two groups, the liver dysfunction group and the control group (normal liver function). The clinical data were compared between the two groups to analyze the risk factors for eltrombopag-induced liver dysfunction.

Definition of liver dysfunction: The severity of the elevation in ALT, aspartate aminotransferase (AST), alkaline phosphatase (ALP), gamma-glutamyl transferase (GGT), and TBIL was evaluated according to the 2017 National Cancer Institute Common Terminology Criteria for Adverse Events (CTCAE v5.0) (9). For each patient, the highest severity of the five biomarkers was used for 
the analysis. Drug-induced liver injury in patients were further classified and their severity evaluated according to Expert panel consensus statement on prevention and standardized treatment of drug-induced liver injury in patients with blood diseases [2016] (10). At our hospital, the reference ranges were ALT 0-50 U/L (male) and 0-35 U/L (female), AST 0-50 U/L (male) and 0-35 U/L (female), ALP 30-120 U/L, GGT 8-57 U/L, and TBIL 0-26 $\mu \mathrm{mol} / \mathrm{L}$ (male) and 0-21 $\mathrm{mol} / \mathrm{L}$ (female).

\section{Statistical analysis}

Descriptive analyses were used to analyze demographics and clinical characteristics. Excel was used to set up a database. SPSS v22.0 was used for data processing and analysis. Count data were analyzed with the $\chi^{2}$ test or Fisher's exact test. Normally distributed measurement data are expressed as $\bar{x} \pm s$ and were analyzed with the two-tailed $t$-test. Nonnormally distributed measurement data are expressed as median $(\mathrm{Q} 1, \mathrm{Q} 3)$ and were analyzed with the nonparametric Wilcoxon rank-sum test. $\mathrm{P}<0.05$ was considered statistically significant.

\section{Results}

\section{General information}

A total of 85 ITP inpatients who met the inclusion criteria were included in this study, including 28 men $(32.9 \%)$ and 57 women $(67.1 \%)$ aged $44 \pm 17$ years $(12-17$ years, $\mathrm{n}=7 ; 19-30$ years, $\mathrm{n}=12 ; 31-40$ years, $\mathrm{n}=16 ; 41-50$ years, $\mathrm{n}=18 ; 51-60$ years, $\mathrm{n}=18 ; 61-70$ years, $\mathrm{n}=10 ; 71-80$ years, $\mathrm{n}=4$ ) with a body mass index (BMI) of $25.1 \pm 3.57 \mathrm{~kg} / \mathrm{m}^{2}$. All the patients were receiving eltrombopag as secondline treatment following failure of first-line treatment. As for comorbidities, 22 patients $(25.9 \%)$ had hypertension, 10 patients (11.8\%) had type 2 diabetes, 12 patients (14.1\%) had noninflammatory hepatobiliary diseases (gallbladder stones, gallbladder polyps, liver cysts, liver fatty), and 16 patients $(18.8 \%)$ had anemia due to iron deficiency.

\section{Eltrombopag dosing and concomitant medications}

The initial dose of eltrombopag was $50 \mathrm{mg} /$ day in 27 patients $(31.8 \%)$ and $75 \mathrm{mg} /$ day in 58 patients $(68.2 \%)$. Treatment time in our hospital was 13 (IQR 9, 18) days. At discharge, the cumulative dose of eltrombopag was 900 (IQR 575, 1,313) $\mathrm{mg}$. Seventy-one patients $(83.5 \%)$ received prophylactic hepatoprotective drugs. During eltrombopag treatment, 32 of 85 patients $(37.7 \%)$ received other drugs that can cause liver dysfunction $(1-2, n=27 ; 3-4, n=5)$.

\section{Liver dysfunction}

After eltrombopag treatment, 19 patients (22.4\%) had liver dysfunction (Table 1). The incidences of elevated ALT, AST, ALP, GGT, and TBIL were $11.8 \%, 10.6 \%, 2.4 \%, 2.4 \%$, and $12.9 \%$, respectively. Fourteen cases $(73.7 \%)$ were grade 1 ; three cases $(15.8 \%)$ were grade 2 ; and two cases $(10.5 \%)$ were grade 3 . Seven patients $(8.2 \%)$ had acute drug-induced hepatocellular injury (mild: $n=6$; moderate: $n=1$ ).

The liver dysfunction group included 6 men and 13 women aged $47 \pm 16$ years; $19-30$ years, $n=2 ; 31-40$ years, $n=5$; $41-50$ years, $n=2 ; 51-60$ years, $n=6 ; 61-70$ years, $n=3 ; 71-$ 80 years, $n=1)$. Eltrombopag treatment time in our hospital was 9 (IQR 6,14) days; the cumulative dose of eltrombopag at discharge was 1,050 (IQR 525, 1,575) $\mathrm{mg}$. Sixteen patients $(84.2 \%)$ received prophylactic hepatoprotective drugs. Seven patients $(36.8 \%)$ used other drugs that can cause liver dysfunction $(1-2, \mathrm{n}=5 ; 3-4, \mathrm{n}=2)$. Due to liver dysfunction, the dose of eltrombopag was reduced and the hepatoprotective treatment intensified in 2 patients $(10.5 \%)$, and eltrombopag was discontinued in 3 patients (15.8\%). At discharge, liver dysfunction did not improve in 10 patients, improved in seven patients, and returned to normal in two patients.

\section{Risk factors of liver dysfunction}

Sex, age, BMI, comorbidities, concomitant medications that can cause liver dysfunction, prophylactic use of hepatoprotective drugs, and initial dose, treatment time, and cumulative dose of eltrombopag were compared between the two groups (Table 2). The results showed that sex, age, BMI, concomitant medications that can cause liver dysfunction, and prophylactic use of hepatoprotective drugs were uncorrelated with liver dysfunction (all $\mathrm{P}>0.05$ ), whereas the comorbidities of type 2 diabetes and noninflammatory hepatobiliary diseases (gallbladder stones, gallbladder polyps, liver cysts, fatty liver) were significantly correlated with liver dysfunction $(\mathrm{P}=0.04, \mathrm{P}=0.023$, respectively).

\section{Discussion}

The introduction of thrombopoietin receptor agonists 
Table 1 Liver dysfunction

\begin{tabular}{|c|c|c|c|c|c|}
\hline Liver dysfunction & Grade 1 & Grade 2 & Grade 3 & Grade 4 & Total \\
\hline AST increased, $\mathrm{n}(\%)$ & $8(9.4)$ & $1(1.2)$ & 0 & 0 & $9(10.6)$ \\
\hline ALP increased, n (\%) & $2(2.4)$ & 0 & 0 & 0 & $2(2.4)$ \\
\hline GGT increased, n, (\%) & 0 & $2(2.4)$ & 0 & 0 & $2(2.4)$ \\
\hline
\end{tabular}

In each patient, more than one indicator could be elevated.

Table 2 Comparison of the clinical characteristics of the liver dysfunction group and the control group (normal liver function) of ITP patients receiving eltrombopag treatment

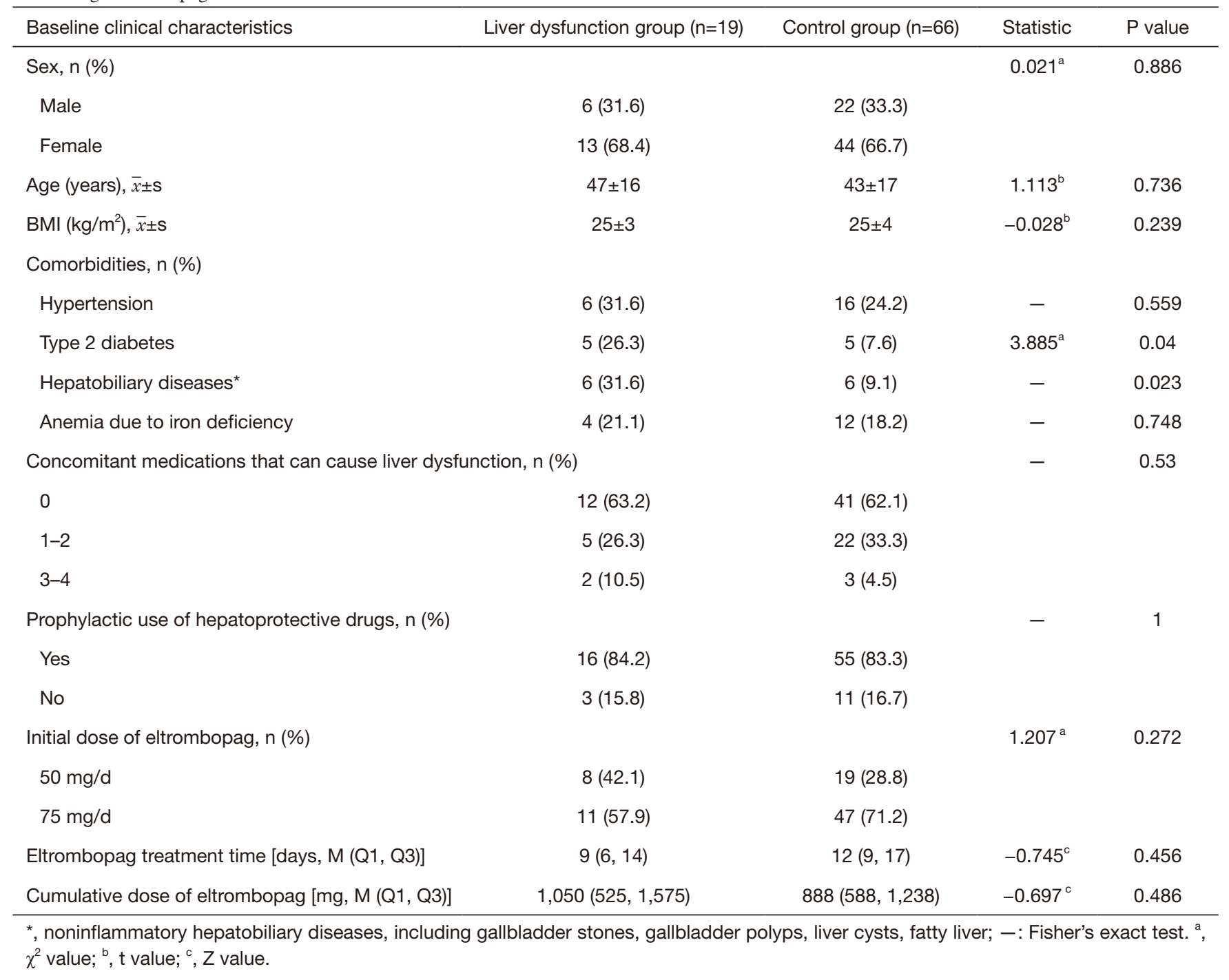


(TPO-RA), such as eltrombopag and romiplostim, has greatly promoted the clinical progress in the treatment of chronic refractory ITP. However, the side effect profile for these new treatments is still under question (11). In this study, we retrospectively analyzed early liver dysfunction in ITP patients who received eltrombopag at our hospital. The incidence of liver dysfunction was $22.4 \%$ (grade 1 : $73.7 \%$; grade 2: $15.8 \%$; grade $3: 10.5 \%$ ). The incidence of drug-induced hepatocellular injury was $8.2 \%$ (mild: $85.7 \%$; moderate: $14.3 \%$ ). Comorbid type 2 diabetes or noninflammatory hepatobiliary disease (gallbladder stones, gallbladder polyps, liver cysts, fatty liver) was correlated with liver dysfunction ( $\mathrm{P}=0.04, \mathrm{P}=0.023$, respectively). In this study, liver dysfunction manifested mostly as mildly elevated ALT or TBIL, which was consistent with earlier reports (7,12-13). Liver dysfunction, mostly mild and reversible after treatment withdrawal, has been reported in many studies, and its incidence is high. Therefore, patients should be closely monitored for serious or potentially lifethreatening liver toxicity. Before treatment, clinicians should explain the risks and precautions and instruct patients to monitor their liver function after discharge. Patients should seek medical attention immediately if they have any abnormal signs or symptoms to minimize the chance of treatment failure due to treatment withdrawal on their own.

The pharmacokinetic profile of eltrombopag varies greatly between different populations. In healthy Japanese volunteers, eltrombopag exposure is twice as high as that of healthy Caucasian volunteers. Moreover, eltrombopag exposure is approximately $87 \%$ higher in East Asian ITP patients than in non-Asian ITP patients (14). In the US and Europe, the recommended initial dose of eltrombopag for the treatment of ITP is $50 \mathrm{mg} /$ day, while Chinese package insert recommends $25 \mathrm{mg}$ /day. A multicenter phase III study in Chinese adults with chronic ITP (7) showed that the dose of eltrombopag averaged at $47.7 \mathrm{mg} /$ day. In this study, the initial dose was 50 or $75 \mathrm{mg} /$ day, and the dose chosen was not correlated with liver dysfunction. The cumulative dose of eltrombopag was also uncorrelated with liver dysfunction. This may be due to the short timeframe of this study, because we only analyzed inpatient data, not follow-up data. It was reported that a 3 -year-old patient with chronic ITP developed acute liver failure due to eltrombopag toxicity (15). A very high eltrombopag plasma concentration was found despite taking the standard drug dosage, and allelic variations that are involved in drug metabolism [CYP2C8 and UDP glucuronosyltransferase
(UGT) 1A1 (UGT1A1)] were carried in this patient. Eltrombopag is extensively metabolized through oxidation by cytochrome P450 (CYP) enzymes, cleavage, and conjugation with glucuronic acid, glutathione, or cysteine. Acute liver failure induced by eltrombopag may be caused by an impaired elimination of the drug due to genetic variants involved in the major pharmacokinetic pathways, leading to the high plasma eltrombopag accumulation in the body. Further research is needed to investigate the proper dose of eltrombopag in Chinese populations and different age groups and the relationships between the plasma concentration or pharmacogenetic of eltrombopag and its safety and efficacy (16).

This was a retrospective study that only analyzed inpatient data of a small sample, which limitations may have resulted in an analysis bias. Large, prospective, randomized, controlled, real-world studies are needed to investigate the mechanism and risk factors of eltrombopag-induced liver dysfunction and drug-induced liver injury.

In summary, ITP patients are at risk for liver dysfunction (mostly mild) after eltrombopag treatment, especially in patients with comorbid type 2 diabetes or hepatobiliary diseases such as gallbladder stones, gallbladder polyps, liver cysts, and fatty liver. To best treat eltrombopaginduced liver dysfunction, early identification of high-risk patients, regular monitoring, early detection, and rational interventions are key.

\section{Acknowledgments}

Funding: None.

\section{Footnote}

Reporting Checklist: We present the following article in accordance with the STROBE reporting checklist. Available at http://dx.doi.org/10.21037/apm-21-1067

Data Sharing Statement: Available at http://dx.doi. org/10.21037/apm-21-1067

Conflicts of Interest: Both authors have completed the ICMJE uniform disclosure form (available at http://dx.doi. org/10.21037/apm-21-1067). The authors have no conflicts of interest to declare.

Ethical Statement: The authors are accountable for all aspects of the work in ensuring that questions related 
to the accuracy or integrity of any part of the work are appropriately investigated and resolved. The research protocol was approved by the Ethics Committee, Institute of Hematology and Blood Diseases Hospital, Chinese Academy of Medical Sciences (HG2021008-EC-1). All procedures performed in this study involving human participants were in accordance with the Declaration of Helsinki (as revised in 2013). Individual consent for this retrospective analysis was waived.

Open Access Statement: This is an Open Access article distributed in accordance with the Creative Commons Attribution-NonCommercial-NoDerivs 4.0 International License (CC BY-NC-ND 4.0), which permits the noncommercial replication and distribution of the article with the strict proviso that no changes or edits are made and the original work is properly cited (including links to both the formal publication through the relevant DOI and the license). See: https://creativecommons.org/licenses/by-nc-nd/4.0/.

\section{References}

1. Thrombosis and Hemostasis Group, Chinese Society of Hematology, Chinese Medical Association. Chinese guideline on the diagnosis and management of adult primary immune thrombocytopenia (version 2020). Zhonghua Xue Ye Xue Za Zhi 2020;41:617-23.

2. Bidika E, Fayyaz H, Salib M, et al. Romiplostim and Eltrombopag in Immune Thrombocytopenia as a SecondLine Treatment. Cureus 2020;12:e9920.

3. Kuter DJ. New thrombopoietic growth factors. Blood 2007;109:4607-16.

4. Chen M, Fang JP, Zhou CX, et al. Efficacy and safety of eltrombopag in the treatment of Chinese children with chronic immune thrombocytopenia. Hematology 2021;26:31-6.

5. Ptushkin VV, Vinogradova OY, Pankrashkina MM, et al. Thrombopoietin Receptor Agonists in the Treatment of Chronic Resistant Primary Immune Thrombocytopenia: Efficacy and Safety Data in Real Clinical Practice. Ter Arkh 2018;90:70-6.

6. Saleh MN, Bussel JB, Cheng G, et al. Safety and efficacy of eltrombopag for treatment of chronic immune thrombocytopenia: results of the long-term, open-label EXTEND study. Blood 2013;121:537-45.

7. Liu X, Hou M, Li J, et al. Efficacy and safety of eltrombopag in Chinese patients with chronic immune thrombocytopenia: stage 2 results from a multicenter phase III study. Platelets 2020. [Epub ahead of print]. doi: 10.1080/09537104.2020.1847267.

8. Cheng G. Eltrombopag, a thrombopoietin- receptor agonist in the treatment of adult chronic immune thrombocytopenia: a review of the efficacy and safety profile. Ther Adv Hematol 2012;3:155-64.

9. National Cancer Institute. Common terminology criteria for adverse events (CTCAE) v5.0. (2017-11-27) [2021-2-27]. Available online: https://ctep.cancer.gov/ protocolDevelopment/electronic_applications/ctc.html

10. Chinese Society of Clinical Oncology (CSCO); Chinese Society of Hematology (CSH); Union for China Lymphoma Investigators (UCLI). Expert panel consensus statement on prevention and standardized treatment of drug-induced liver injury in patients with blood diseases (2016). Zhonghua Xue Ye Xue Za Zhi 2016;37:441-52.

11. Samson M, Fraser W, Lebowitz D. Treatments for Primary Immune Thrombocytopenia: A Review. Cureus 2019;11:e5849.

12. Yang R, Li J, Jin J, et al. Multicentre, randomised phase III study of the efficacy and safety of eltrombopag in Chinese patients with chronic immune thrombocytopenia. $\mathrm{Br} \mathrm{J}$ Haematol 2017;176:101-10.

13. Cheng G, Saleh MN, Marcher C, et al. Eltrombopag for management of chronic immune thrombocytopenia (RAISE): a 6-month, randomised, phase 3 study. Lancet 2011;377:393-402.

14. Tomiyama Y, Miyakawa Y, Okamoto S, et al. A lower starting dose of eltrombopag is efficacious in Japanese patients with previously treated chronic immune thrombocytopenia. J Thromb Haemost 2012;10:799-806.

15. Marano M, Serafinelli J, Cairoli S, et al. EltrombopagInduced Acute Liver Failure in a Pediatric Patient: A Pharmacokinetic and Pharmacogenetic Analysis. Ther Drug Monit 2018;40:386-8.

16. Zuo W, Zhang B, Ruan J, et al. Correlation of the Plasma Concentration of Eltrombopag With Efficacy in the Treatment of Refractory Aplastic Anemia: A Single-Centre Study in China. Front Pharmacol 2020;11:582625.

Cite this article as: Zhang $\mathrm{P}$, Miao W. Eltrombopaginduced liver dysfunction during the treatment of immune thrombocytopenia and its risk factors. Ann Palliat Med 2021;10(6):6419-6424. doi: 10.21037/apm-21-1067 